\title{
OAS1 genotype linked to multiple sclerosis severity
}

A research team based in Dublin, Ireland has provided new evidence that a single nucleotide polymorphism (SNP) in the oligoadenylate synthetase 1 (OAS1) gene, the product of which has a pivotal role in the innate immune response to viral infection, influences multiple sclerosis (MS) susceptibility and disease severity.

The SNP is situated at the exon 7 splice-acceptor site of OAS1, and gives rise to three possible genotypes: AA, GG and AG. The new study, conducted by Margaret O'Brien and colleagues at St Vincent's University Hospital, indicates that individuals with the AA genotype are more susceptible to developing MS than those with either of the other two genotypes. The GG genotype, on the other hand, seems to confer a protective effect against increased disease activity in patients with MS.

First, O'Brien et al. examined the distributions of the different OAS1 alleles and genotypes in 401 patients with MS and 394 healthy controls. They found that the A allele occurred more frequently in the MS cohort than in the control group (63\% versus $57 \%$ ), whereas the reverse was true for the G allele (37\%

\section{1 ...the AA genotype occurred more frequently in the MS cohort than in the control group... 77}

versus $43 \%$ ). In patients with MS, the genotype frequencies were $33 \%, 61 \%$ and $6 \%$ for AA, AG and GG, respectively, compared with $30 \%, 53 \%$ and $17 \%$ in healthy controls.

Next, the researchers measured the frequencies of the different OAS1 genotypes in relation to MS disease activity and interferon (IFN)- $\beta$ treatment. Of 108 patients receiving IFN- $\beta$ who were judged to have little or no disease activity, 32\% had the AA genotype, $61 \%$ had the AG genotype, and 7\% had the GG genotype. For 53 patients who showed disease activity despite IFN- $\beta$ treatment, the respective figures were $51 \%, 47 \%$ and $2 \%$. In a further group of 65 patients who had highly active relapsing-remitting MS, the genotype frequencies were $34 \%$ for AA, $66 \%$ for $A G$ and $0 \%$ for $G G$.

O'Brien et al. also correlated the three OAS1 genotypes with the time to first relapse on IFN- $\beta$ therapy. They found that the median time to first relapse was 24 months in patients with the AA genotype, compared with 33 months in those with the AG or GG genotype.

OAS1 is activated by type 1 IFNs, and its protein product is an important component of the endogenous IFN antiviral pathway. The A allele encodes a truncated form of OAS1 with low enzymatic activity. Viral infections have previously been implicated in MS relapses, and O'Brien et al. suggest that impairment of antiviral activity resulting from OAS1 truncation could account for the shorter time to first relapse in patients with the AA genotype.

The researchers recommend that all patients who are about to commence treatment for MS should be genotyped at the OAS1 locus. Patients with the AA genotype could then be monitored closely for disease activity and promptly switched to a different treatment regimen if they show signs of a poor response to the first-line therapy.

Heather Wood

Original article 0'Brien, M. et al. OAS1: a multiple sclerosis susceptibility gene that influences disease severity. Neurology 75, 411-418 (2010) 